\title{
Perspectiva de género, desafíos para su inclusión en las políticas públicas
}

Carmen Echeverría Cabrera

\section{Resumen}

Alcanzar la igualdad sustantiva entre mujeres y hombres en México conlleva limitaciones y obstáculos de carácter cultural e institucional. Entre ellos está la resistencia a los cambios de paradigma de lo que significa ser mujer y ser hombre, expresados en los roles y estereotipos de género. El diseño e implementación de las políticas públicas tienen grandes desafíos para erradicar la violencia contra las mujeres, como máxima expresión de las desigualdades por razones de género.

Palabras clave: clave: perspectiva de género, políticas públicas, igualdad de género, violencia de género.

\section{GeNDER PERSPECTIVE, CHALLENGES fOR ITS INCLUSION IN PUBLIC POLICIES}

\begin{abstract}
Reaching a substantive equality between women and men in Mexico carries cultural and institutional limitations and obstacles. Among them, the resistance to paradigm shifts regarding being a women and men, expressed in gender roles and stereotypes. The design and implementation of public politics face big challenges to eradicate the violence against women as a maximum expression of the inequalities for gender-based reasons.
\end{abstract}

Keywords: gender perspective, public politics, gender equality, gender-based violence. 


\section{Carmen Echeverría Cabrera}

Psicoterapeuta e integrante del Centro de salud mental y género. Licenciada en pedagogía, Facultad de Filosofía y Letras de la Universidad Nacional Autónoma de México (UnAM). Maestra en Investigación y Desarrollo de la Educación por la Universidad Iberoamericana, campus Santa Fe, Ciudad de México. Máster en gobernabilidad y desarrollo humano por la Universidad Oberta de Cataluña en España, y especialidad en el Programa Superior en violencia de género: prevención, detección y atención del Centro Universitario La Salle, Universidad Autónoma de Madrid.

Consultora en materia de políticas públicas para la prevención y atención de la violencia de género en contra de las mujeres y la igualdad entre mujeres y hombres. Ha coordinado estudios con enfoque de género, derechos humanos, e interculturalidad. Ha dirigido la construcción de diversos modelos de intervención pública y social para la infancia y juventud en situación de calle (Modelo Matlapa); Refugios para mujeres, sus hijas e hijos en situación de violencia familiar y sexual; Prevención y atención de la violencia, especialmente contra las mujeres en Ciudad Juárez, Chihuahua y el Modelo de trabajo a nivel comunitario para la prevención de la violencia y el ejercicio de los derechos de las mujeres rarámuri con enfoque intercultural. En el Gobierno federal fundó y se desempeñó como Coordinadora Nacional del Programa de apoyo a las Instancias de Mujeres en las entidades federativas, PAImEF. También ha realizado evaluaciones al Programa de Fortalecimiento a la Transversalización de la perspectiva de género, del InMuJeres y del Sistema Nacional de prevención, atención, sanción y erradicación de la violencia contra las mujeres para la conAvim, dependiente de la Secretaría de Gobernación. 


\section{Introducción}

Este artículo pretende dar cuenta de algunos de los obstáculos y desafíos en el diseño e implementación de políticas públicas con perspectiva de género, presentes en las instituciones públicas que diseñan y operan programas de prevención y atención de la violencia contra las mujeres, o con la intención de transversalizar la perspectiva de género en la administración pública. Se parte de la consideración de que los roles y estereotipos de género, con frecuencia, están interiorizados en las y los diseñadores y operadores de esas instituciones. ${ }^{1}$

\section{Obstáculos en el diseño e implementación de las políticas públicas en materia de prevención y atención de la violencia contra las mujeres}

Lo expuesto en este trabajo tiene como base mi experiencia como funcionaria de la administración pública federal en el diseño e implementación de políticas públicas relacionadas con la prevención y atención de la violencia contra las mujeres, particularmente en el Programa de Apoyo a las Instancias de Mujeres en las Entidades Federativas (PAIMEF) y de evaluar el Programa de Fortalecimiento a Transversalidad de la Perspectiva de Género (PFTPG).

${ }^{2}$ La perspectiva de género es una herramienta conceptual que busca mostrar que las diferencias entre mujeres y hombres se dan no sólo por su determinación

biológica, sino también por las diferencias culturales, construidas históricamente, asignadas a los seres humanos.

Alude a una normatividad establecida por el patriarcado y la heterosexualidad, lo que le da soporte al sistema social y en donde el género (masculino - femenino) establece una jerarquización de espacios, recursos materiales, económicos, políticos, públicos e ideológicos; su legitimación se da a través de la religión, la política, la filosofía y la historia.
En el siglo xx, cuando el discurso sobre el desarrollo empezó a estar presente en las políticas de los países industrializados, prácticamente no se prestó atención a las desigualdades existentes entre hombres y mujeres. De acuerdo con los primeros estudios sobre el impacto de la modernización, las mujeres no estuvieron presentes en las acciones ni en los beneficios del desarrollo, sino que hasta veían empeorar su estatus familiar y social. Ante ello, las feministas buscaron hacer visible el aporte de las mujeres al desarrollo, y lograron demostrar que los planificadores de éste habían actuado utilizando estereotipos sobre las mujeres y su papel productivo (Weis, 2015).

En mi experiencia en el diseño e implementación de políticas públicas con perspectiva de género ${ }^{2}$, particularmente en materia de prevención y atención de la violencia contra las mujeres, he tenido la oportunidad de percatarme de algunos obstáculos, objetivos y subjetivos, que influyen en el diseño, implementación y, eventualmente, en la evaluación de las políticas públicas. Todos ellos traen como consecuencia la normalización de la violencia contra las mujeres, la impunidad, la injusticia, así como la profundización de las desigualdades. A continuación, listo los factores que limitan el diseño e implementación de las políticas públicas en materia de prevención y atención de la violencia contra las mujeres.

\section{Factores objetivos limitantes}

Debilidad institucional para prevenir y atender la violencia contra las mujeres en los tres órdenes de gobierno:

- Visión poco clara referente a la transversalización del tema. Acciones de sensibilización fragmentadas al interior de las instituciones en las que el personal directivo es el menos participante.

- Desarticulación interinstitucional e intersectorial para impulsar la agenda en la materia. 
- Deficiente vinculación entre actores públicos, sociales y académicos especializados en la materia.

- Rotación del personal debido a la violencia institucional. Falta de uso de procedimientos que favorezcan el autocuidado ${ }^{3}$ y nulas condiciones para realizar el trabajo bajo esquemas de seguridad social.

- Retraso, escasez y, frecuentemente, nulos recursos económicos para la realización de los proyectos sensibles al género, sobre todo en los municipios con la Declaratoria de Alerta de Violencia de Género.

- Acciones de prevención deficientes y desvinculadas de las necesidades específicas de la población a nivel local.

- Deficientes o inexistentes estrategias de monitoreo, seguimiento y evaluación de las acciones realizadas.

- Escasa sistematización en torno a los factores críticos de éxito y de fracaso en la atención.

- Escasa y deficiente oferta institucional de servicios especializados, sobre todo en municipios lejanos a las capitales de los estados.

- Reiteradas omisiones al cumplimiento normativo en relación con la garantía de los derechos de las mujeres a una vida libre de violencia.

- La violencia feminicida y su impunidad es una muestra de la dificultad para enfrentar procesos de cambio en el paradigma de la igualdad entre mujeres y hombres y en todos los aspectos de nuestra vida. Esta violencia se repite debido a una deficiencia del Estado para brindar protección y justicia a las mujeres.

\section{Factores subjetivos limitantes}

- Una tendencia a universalizar lo que significa ser hombre o mujer, madre, padre, hijo, hija, lo que es una pareja, una familia o las diferentes familias. Por lo tanto, se tiende a descontextualizarlos de la cultura o de la sociedad.

${ }^{3}$ Véase Daskal, A. M. (1994). Permiso para quererme. Reflexiones sobre la autoestima femenina. Paidós. Argentina.

${ }^{4}$ Para consultar conceptos básicos de género, véase a Mercedes Barquet y Alejandra Benítez (2012): La transversalización de la perspectiva de género: una estrategia para avanzar a la igualdad.
- Está presente un prejuicio de que atender las necesidades e intereses estratégicos de las mujeres 4 tendrá como resultado un mayor enfrentamiento entre hombres y mujeres y, por lo tanto, es destructor de la pareja o de la familia.

Los factores objetivos y subjetivos se entremezclan y, lamentablemente, prevalecen, a pesar de los esfuerzos importantes que ha habido por incorporar y operativizar la perspectiva de género, principalmente en las reglas de operación y lineamientos de algunos programas federales. En la práctica institucional, rara vez se aplican adecuadamente. 


\section{Desafíos para la inclusión de la perspectiva de género en las políticas públicas de prevención y atención de la violencia contra las mujeres}

Ante la escalada de violencia en nuestro país, el Estado mexicano reconoció la violencia contra las mujeres como un asunto de interés público y materia de política pública hace tan sólo trece años, con la publicación de la Ley General de Acceso de las Mujeres a una Vida Libre de Violencia (LGAMVLV), el $1^{\circ}$ de febrero de 2007. En ella se define la violencia contra las mujeres como "cualquier acción u omisión, basada en su género, que les cause daño o sufrimiento psicológico, físico, patrimonial, económico, sexual o la muerte tanto en el ámbito privado como en el público".

Los tipos de violencia que contempla la LGAMvLv son: emocional, física, económica, patrimonial y sexual. Asimismo, considera los ámbitos en que ocurre: escolar, laboral, docente, comunitario, institucional, familiar y feminicida. Es importante resaltar que la violencia contra las mujeres, hasta hace pocos años, había sido considerada sólo en el ámbito privado.

Por su parte, la Encuesta Nacional sobre la Dinámica de las Relaciones en los Hogares (ENDIREH), en su última edición (INEGI, 2016), destaca que en México 66.1\% de las mujeres a partir de los 15 años reconoció haber experimentado por lo menos un incidente de violencia a lo largo de su vida: 49\% ha sufrido violencia emocional y 29\% ha padecido violencia económica, patrimonial o discriminación en el trabajo.

Pese a los grandes esfuerzos del movimiento de mujeres y de la sociedad civil organizada, la violencia de género en nuestra sociedad aún se encuentra normalizada y, por lo tanto, es aceptada y mantenida en secreto, por lo que las víctimas se asumen en el desamparo. Cotidianamente se escuchan testimonios de la población femenina ${ }^{5}$ en los que se vive la violencia en un grado extremo, como el caso siguiente:

${ }^{5}$ Testimonios que pueden ser escuchados con la persona confidente de la víctima, en centros de atención para las mujeres, refugios y en el mejor de los casos en sesión de terapia, donde la familia de la víctima regularmente desconoce lo que le vive esta persona.

${ }^{6}$ Ante el confinamiento por la emergencia sanitaria del covid-19, las mujeres, niñas y niños quedan

bajo una mayor exposición a la violencia, incrementándose el feminicidio. Véase covid-19 y su impacto en la violencia contra las mujeres y niñas.
[...] a mí me pegaron, me quemaron, me violaron, me dejaron en descampado, me asfixiaron, me insultaron, me humillaron, y yo soy, siembre digo, básicamente, el ejemplo a no seguir. Yo nunca me atreví a denunciar [...] (Marroquí, 2019). ${ }^{6}$

Este testimonio da cuenta de varios de los obstáculos que las mujeres enfrentan para erradicar la violencia de sus vidas. Algunos de éstos tienen hondas raíces en los roles y estereotipos de género, construidos cultural e históricamente, los cuales moldean el ser y el hacer de mujeres y hombres. En ambos casos, se considera que la esencia femenina o masculina son universales; a los hombres, por su "naturaleza", les corresponde hacer determinadas actividades en la sociedad (roles), tales como proveer, garantizar el dinero en la casa, estar a cargo de una familia, ser audaces, fuertes, dominantes, contar con voluntad. Dependiendo de su perfil psicológico y el contexto en que se desarrollen, incluso podrían tender a ser violentos, al utilizar esos espacios de poder y de privilegios. 
A las mujeres, la sociedad las prepara para desarrollar un rol en donde las tareas del hogar son su responsabilidad, así como del cuidado de familiares, incluyendo a las y los enfermos o personas con alguna discapacidad. En su manera de ser, está el ser sumisas, calladas, cálidas, y estar acompañadas, principalmente de un hombre. Difícilmente se las educa para prepararse y desarrollar cualidades que las conviertan en una persona autosuficiente, independiente y autónoma.

Los estereotipos de género, entonces, son creencias, imágenes e ideas generalizadoras y socialmente compartidas que se consideran propiamente femeninas o masculinas, y que guían la formación de ciertas expectativas, evaluaciones y formas de ser en torno a la conducta esperada de los individuos (Freidenberg, 2018). Sin embargo, son cambiantes en el tiempo y renegociados entre generaciones (Weis, 2015).

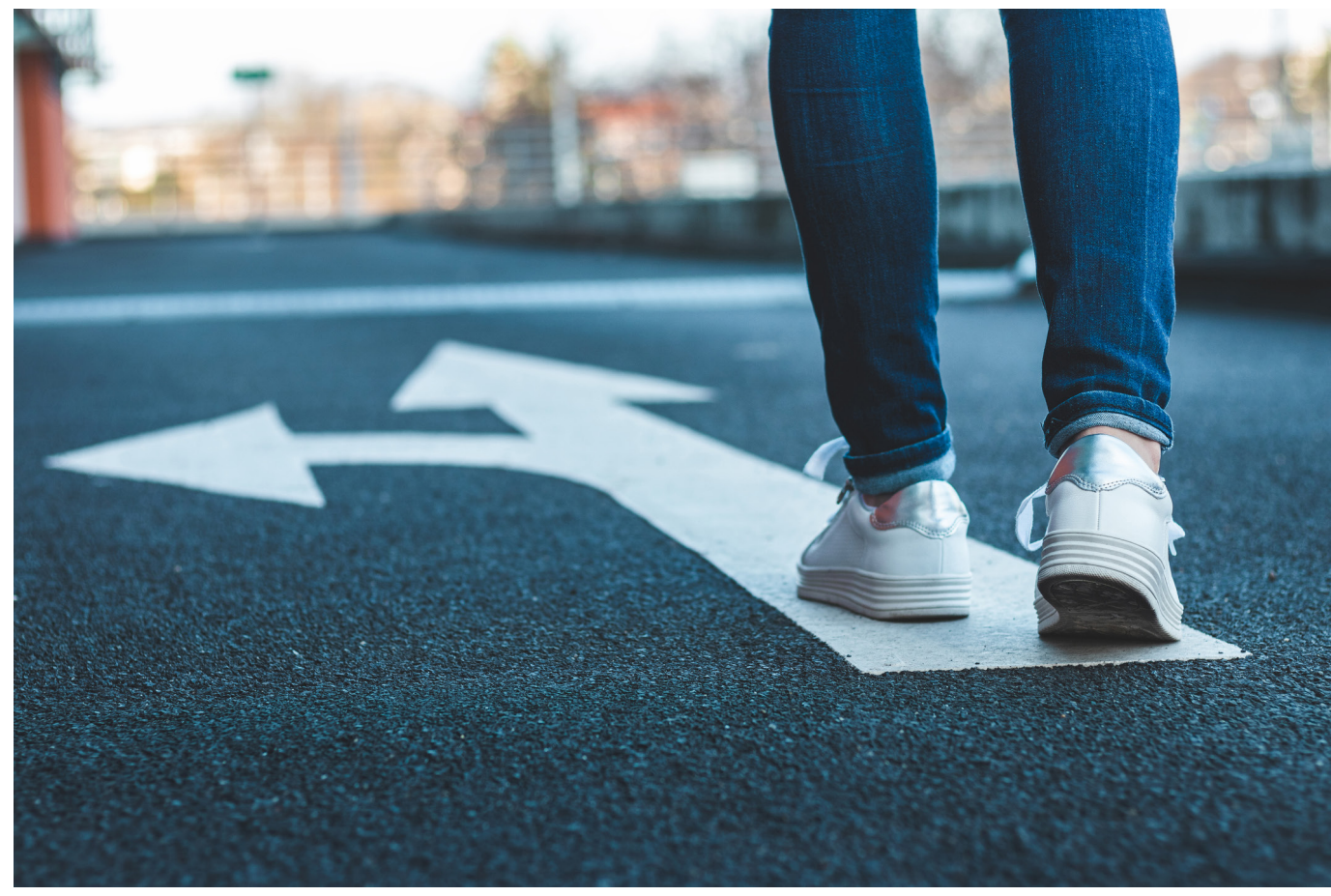

Este conjunto de creencias se practica y se reproduce en un sistema patriarcal, donde existe la supremacía, poder y dominio de los hombres sobre las mujeres, la cual se ha institucionalizado y generalizado, por lo que se extiende a todas las relaciones sociales entre mujeres y hombres, en los distintos ámbitos de interacción social (Facio y Fries, 2005). Este sistema, al marcar profundas desigualdades, vulnera a las mujeres (Hirigoyen, 2014, p.40) y las violenta.

La violencia por razones de género se presenta en todos los grupos sociales y en todos los niveles socioculturales. Las mujeres pueden ser víctimas de violencia de género durante todo su ciclo de vida y en todos los ámbitos en los que se desenvuelven. Esto trastoca las generaciones subsecuentes, quienes reproducen los mismos estereotipos, tanto en los estilos de crianza como de 
relación, lo que va perpetuando las relaciones o vínculos matizados por la violencia. Bourdieu (2000) ha denominado a esta situación violencia simbólica, en donde la persona dominada adopta para sí, sin saberlo, el punto de vista de la persona dominante, adaptando, en cierto modo, para evaluarse, la lógica del prejuicio desfavorable.

Esta dominación tiene como resultado la desigualdad ${ }^{7}$ y la violencia es la máxima expresión del desequilibrio de poder que hay en las relaciones entre mujeres y hombres. La desigualdad se expresa a través de las brechas de género, las cuales reflejan la diferencia existente entre los sexos respecto a las oportunidades de acceso y control de recursos económicos, sociales, culturales y políticos, entre otros.

En las instituciones públicas se puede percibir que los estereotipos de género continúan influyendo de manera significativa en las decisiones políticas, que son trascendentales, sobre cómo deberán atenderse las problemáticas relacionadas con las desigualdades sociales por razones de género. Esto obedece a que las instituciones están conformadas por personas, por lo que la construcción de un problema público puede desvelar la posición -respecto al género- que guardan quienes toman las decisiones sobre los recursos públicos, así como sobre la forma en la que se entienden los problemas sociales y las intervenciones públicas que se eligen para atenderlos.

Para enfrentar esta situación, las políticas públicas con perspectiva de género se han orientado, grosso modo, hacia lo siguiente:

- Estudios para identificar las principales brechas de desigualdad entre mujeres y hombres.

- Desarrollo de un marco programático para la atención de diversos tipos de violencia con diferentes poblaciones, violencia obstétrica, feminicida, familiar, laboral, institucional, entre otras.

- Elaboración de manuales para el trabajo con diferentes poblaciones: niñas, niños, adolescentes, jóvenes, personas adultas mayores.

- Creación y fortalecimientos de refugios para mujeres en situación de extrema violencia.

- Creación y fortalecimiento de Casas de la Mujer indígena. ${ }^{8}$

7 Véase Carbonell, M. (2009). La igualdad insuficiente. II-UNAM/ CNDH/CONAPRED.

${ }^{8}$ A partir de 2020, la asignación del presupuesto para este programa está en riesgo.
- Campañas de comunicación social para la prevención de la violencia.

- Atención directa a las mujeres que viven violencia a través de la orientación jurídica, psicológica y de trabajo social.

- Capacitación al funcionariado de las distintas dependencias que conforman los sistemas estatales para prevenir, atender, sancionar y erradicar la violencia contra las mujeres. 


\section{Conclusiones}

Hay progresos reales en las condiciones de las mujeres, así como una creciente visibilización de la violencia contra las mujeres por razones de género. Se han abierto espacios, aún insuficientes, para la atención externa desde una perspectiva jurídica, psicoterapéutica y de asistencia, refugios, redes de solidaridad. Asimismo, hay un marco legal, aún con muchas limitaciones, para la procuración de justicia y una creciente apertura en los medios de comunicación para hablar de la violencia.

Todavía hay mucho por hacer para evidenciar a la violencia de género como una de las barreras que restringen el avance de las mujeres hacia su independencia y autonomía, así como el acceso a espacios de decisión, tanto en la esfera privada como en la pública.

La variable de género, al ser incluida en las políticas públicas, debe considerarse como una categoría de poder, lo cual es un principio asociado con valoraciones éticas, políticas y filosóficas sobre el ideal de justicia entre las mujeres y los hombres, justicia que debe ser orientada a transformar y superar las desigualdades que, argumentando la diferencia sexual, se han construido a lo largo de la historia.

El diseño y la operación de las políticas implica revisar la perspectiva conceptual y metodológica que acompaña la definición del actuar gubernamental. La construcción de un problema público puede desvelar la posición que guardan quienes toman las decisiones sobre los recursos públicos, sobre las formas en las que se entienden los problemas sociales, así como sobre la determinación, clasificación y selección de causas que se propone abordar y los cómos.

Los obstáculos y desafíos de las políticas públicas con perspectiva de género adoptadas y mencionadas anteriormente coexisten con el sistema patriarcal antes mencionado, lo que dificulta de forma casi invisible los alcances de los programas y acciones.

\section{Referencias}

* Barquet, M. y Benítez, A. (2012). La transversalización de la perspectiva de género: una estrategia para avanzar a la igualdad. Suprema Corte de Justicia de la Nación, Tribunal Electoral del Poder Judicial de la Federación, Instituto Electoral del Distrito Federal. http://portal.iedf.org.mx/biblioteca/descargasC.php?id=75

* Bourdieu, P. (2000). La dominación masculina [Trad. Joaquín Jordá]. Anagrama.

* Carbonell, M. (2009). La igualdad insuficiente. IIJ-UNAM/CNDH/CONAPRED.

* Daskal, A. M. (1994). Permiso para quererme. Reflexiones sobre la autoestima femenina. Paidós. 
* Facio, A. y Fries, L. (2005). Feminismo, género y patriarcado. Academia. Revista sobre enseñanza del derecho de Buenos Aires, 3(6), Primavera 2005.

* Freidenberg, Flavia. (2018). "Ellas también saben": estereotipos de género, resistencias a la inclusión y estrategias para feminizar la política [entrevista realizada y publicada por el periódico El País]. http://www.revista.ibd.senado.gob. mx/index.php/PluralidadyConsenso/article/viewFile/521/482

* Hirigoyen, M-F. (2014). Mujeres maltratadas. Los mecanismos de la violencia en la pareja [Trad. Gemma Andújar Moreno]. Paidós.

- Instituto Nacional de Estadística y Geografía (INEGI). (2016). Encuesta Nacional sobre la Dinámica de las relaciones en los hogares (ENDIREH). https://www.inegi.org.mx/ programas/endireh/2016/

* Marroquí, M. (2019, 25 de noviembre). La sociedad aún no entiende lo que es la violencia de género, educadora social [video]. YouTube. https://www.youtube.com/ watch? $v=i u b i K W r B c B M$

* Diario Oficial de la Federación (DOF). (2007, 1 de febrero). Ley General de Acceso de las Mujeres a una Vida Libre de Violencia. http://www.dof.gob.mx/nota detalle. php?codigo $=4961209 \&$ fecha $=01 / 02 / 2007$

* Weis, A. R. (2015). La perspectiva de género en la cooperación internacional al desarrollo. Conceptos básicos y buenas prácticas. Deutsche Gesellschaft für Internationale Zusammenarbeit (GIZ); Proyecto de cooperación para el fortalecimiento institucional de la AMEXCID. Agencia de la GIz en México. https:// www.giz.de/en/downloads/giz2015-es-La perspectiva de genero en la cooperacion internacional al desarrollo.pdf

\section{Cómo citar el artículo}

* Echeverría Cabrera, Carmen. (2020). Perspectiva de género, desafíos para su inclusión en las políticas públicas. Revista Digital Universitaria (RDU), 21(4) julioagosto. Dol: http://doi.org/10.22201/cuaieed.16076079e.2020.21.4.10

Recepción: 16/05/2020. Aceptación: 29/05/2020 